\section{Atenção à saúde bucal no Parque Indígena do Xingu, Brasil, no período de 2004-2013: um olhar a partir de indicadores de avaliação}

\author{
Oral health care in the Xingu Indigenous Park, \\ Brazil, from 2004 to 2013: an analysis based \\ on evaluation indicators
}

\section{Atención a la salud bucal en el Parque Indígena de Xingu, Brasil, durante el período de 2004 a 2013: una observación a partir de indicadores de evaluación}

Pablo Natanael Lemos 1,2 Douglas Antônio Rodrigues 2 Paulo Frazão 1

Lucila Brandão Hirooka 3 Alana Cristina Guisilini 4 Paulo Capel Narvai 1

doi: 10.1590/0102-311X00079317

\title{
Resumo
}

A Politica Nacional de Saúde Bucal redefiniu em 2011 seu modelo de atenção para os povos indígenas, preconizando o subsídio da epidemiologia e o acompanhamento do impacto das ações por meio de indicadores adequados. $O$ objetivo deste estudo foi analisar a evolução desses indicadores, propostos pelo Ministério da Saúde, no Parque Indígena do Xingu, Brasil, no período de 2004-2013. Trata-se de pesquisa de abordagem quantitativa, com o uso de dados secundários do Distrito Sanitário Especial Indígena Xingu e do Projeto Xingu, da Universidade Federal de São Paulo. Observou-se cobertura de primeira consulta odontológica programática maior que 60\% em todos os anos analisados, exceto em 2009 e 2010, cujas coberturas foram de 44,7\% e 53, 4\%, respectivamente. $O$ indicador de tratamento odontológico básico concluído apresentou aumento significativo, de 44,9\% para 79,9\%, entre 2006 e 2008. A proporção de exodontias no conjunto dos procedimentos diminuiu de $24,3 \%$ em 2004 para 3,8\% em 2011. A cobertura da média da ação coletiva de escovação dental supervisionada obteve a maior variabilidade $(1,2$ a 23,3\%) no período analisado. O acesso à saúde bucal mostrou boa cobertura e o indicador de tratamento concluído apresentou percentual mais elevado em comparação com outros povos indígenas no mesmo período. O melhor desempenho do indicador de exodontias pode decorrer de mudança no enfoque assistencial possibilitado por parcerias com universidades, ainda que os indicadores de escovação supervisionada indiquem ser necessário priorizar ações preventivas. Mudanças na gestão da saúde indígena, com fragilização ou ausência de parcerias, podem ter influenciado negativamente os indicadores do programa.

Saúde de Populações Indígenas; Saúde Bucal; Indicadores de Serviços;

Indios Sul-Americanos

\author{
Correspondência \\ P. N. Lemos \\ Rua Estado de Israel 113, São Paulo, SP 04022-000, Brasil. \\ pablonlemos@usp.br \\ 1 Faculdade de Saúde Pública, Universidade de São Paulo, São \\ Paulo, Brasil. \\ 2 Escola Paulista de Medicina, Universidade Federal de São \\ Paulo, São Paulo, Brasil. \\ 3 Faculdade de Medicina de Ribeirão Preto, Universidade de São \\ Paulo, Ribeirão Preto, Brasil. \\ ${ }_{4}$ Faculdade de Odontologia de Piracicaba, Universidade \\ Estadual de Campinas, Piracicaba, Brasil.
}




\section{Introdução}

Desde a promulgação da Constituição Federal de 1988, muitas mudanças ocorreram nas políticas públicas voltadas aos povos indígenas no Brasil. A criação do Sistema Único de Saúde (SUS) e posteriormente o Subsistema de Atenção à Saúde Indígena (SASI-SUS) possibilitaram a implementação de ações e programas de saúde nos territórios indígenas. A avaliação desses programas tem sido um importante desafio tanto para a consolidação dos princípios do SUS quanto para a tomada de decisões sobre ajustes ou reformulações de suas propostas iniciais, buscando melhorias nos processos administrativos, políticos e sociais 1 .

A Política Nacional de Saúde Bucal, em 2004, consolidou a atenção à saúde bucal pela reorganização dos serviços de saúde. A atenção à saúde é orientada por um modelo assistencial resultante de um processo histórico contraditório durante o qual diversos atores sociais, setores e instituições interagem sob a lógica de distintos interesses 2 . Assim, como em outras populações, a saúde bucal dos povos indígenas guarda uma estreita relação com determinantes ambientais, culturais, econômicos e biológicos 3,4. Os estudos internacionais sobre a saúde de povos indígenas têm indicado disparidades na oferta de serviços de saúde bucal entre indígenas e não indígenas 5,6. Entretanto, alguns artigos têm relatado experiências e abordagens bem-sucedidas de saúde bucal em populações indígenas como a integração de serviços odontológicos na atenção primária à saúde, programas intersetoriais de promoção de saúde bucal e ações de reconhecimento das práticas tradicionais, especialmente no Canadá, Estados Unidos, Austrália e Nova Zelândia 7,8,9,10,11,12,13.

No Brasil, em 2011, o Ministério da Saúde definiu a reorganização do modelo de atenção em saúde bucal direcionado aos povos indígenas, por meio de ações de controle das doenças bucais e de promoção e recuperação da saúde, planejadas com base em um diagnóstico das condições de saúde-doença, subsidiado pela epidemiologia e informações sobre o território indígena, além do acompanhamento do impacto das ações de saúde bucal por meio de indicadores adequados 14 .

Os indicadores de saúde são instrumentos valiosos para a gestão e avaliação da situação de saúde de uma população. De forma geral, são medidas que trazem informações relevantes sobre determinados atributos do estado de saúde, bem como do desempenho do sistema de saúde, facilitando o monitoramento dos objetivos e metas em saúde 15. As condições de acesso a serviços de saúde refletem as características da oferta desses serviços, podendo facilitar ou dificultar sua utilização pelos indivíduos, de acordo com suas necessidades. A discussão sobre acessibilidade aos serviços de saúde bucal é bastante complexa, necessitando de estudos que contemplem perspectivas, de forma a apreender a realidade localmente observada 16.

Para assegurar a confiança das informações produzidas nos serviços de saúde é preciso monitorar a entrada de dados e os valores dos indicadores, revisando periodicamente a consistência da série histórica. A utilização de sistemas de informação de saúde é uma forma de aproveitamento de dados secundários de coleta continuada. Eles vêm sendo crescentemente empregados na pesquisa e na avaliação em saúde, além de ser um facilitador na tomada de decisão dos profissionais e gestores da saúde 17.

O Parque Indígena do Xingu (PIX) foi a primeira terra indígena reconhecida no país, em 1961. O Projeto Xingu da Escola Paulista de Medicina, Universidade Federal de São Paulo (UNIFESP), desenvolve ações e pesquisas em saúde no PIX desde 1965. Com a criação do SASI-SUS em 1999, o Projeto Xingu foi convidado pelo Departamento de Saúde Indígena da Fundação Nacional de Saúde (FUNASA) e por lideranças indígenas do PIX a fazer convênios para dar início à implementação do Distrito Sanitário Especial Indígena (DSEI) Xingu. Sob coordenação do Projeto Xingu, as ações de saúde bucal passaram a assumir feições programáticas. Porém, a pequena quantidade de dentistas na equipe de saúde e a grande demanda assistencial acumulada ao longo dos anos levou o Projeto Xingu a buscar parcerias para a qualificação da atenção à saúde bucal, na forma de um modelo de atenção pautado na vigilância da saúde. Nesse contexto, a partir de 2003, a Faculdade de Odontologia de Ribeirão Preto, Universidade de São Paulo (FORP-USP) e o Projeto Pro Nativos, financiado pela Colgate, passaram a colaborar na atenção à saúde bucal no Xingu 18 .

Durante a execução dos convênios, problemas estruturais e operacionais da FUNASA geraram descontinuidade dos serviços, fragilidades nos fluxos de financiamentos e falta de monitoramento da execução das ações 19. O impacto no DSEI Xingu foi sentido e, a partir de 2007, as parcerias sofreram 
alterações. A FUNASA interrompeu a celebração de convênio com a FORP-USP e o Projeto Pro Nativos se encerrou em razão do término do financiamento de sua instituição mantenedora. O convênio do Projeto Xingu com a FUNASA sucedeu-se até 2010, quando a gestão do SASI-SUS passou a ser de responsabilidade da Secretaria Especial de Saúde Indígena (SESAI).

A análise da situação de saúde como prática sistemática com auxílio de indicadores apresenta um potencial ainda inexplorado nos diversos níveis dos serviços de saúde, apesar da grande quantidade de dados produzidos rotineiramente. A escolha de indicadores acessíveis, simples, úteis e disponíveis nos sistemas de informação valoriza as bases de dados existentes e indica o seu potencial no apoio à gestão 20 . O uso de indicadores na avaliação de programas de saúde indígena é um requisito para a formulação de novas propostas de intervenção e tomada de decisão, com o intuito de aprimorar os programas e serviços ofertados.

No presente estudo, analisou-se a evolução de indicadores de cobertura de primeira consulta odontológica programática, tratamento odontológico básico concluído, proporção de exodontia em relação aos procedimentos, cobertura média mensal da escovação dental supervisionada do PIX, no período de 2004-2013.

\section{População e métodos}

O PIX localiza-se na região nordeste do Estado do Mato Grosso, na parte sul da Amazônia brasileira, com uma população total de aproximadamente 6 mil pessoas, abrigando 16 etnias. O território estudado se localiza na região central e ao norte do PIX e corresponde às áreas de abrangência dos polos-bases Pavuru, Diauarum e Wawi. No total são 44 aldeias das etnias Kisêdje, Ikpeng, Kaiabi, Trumai, Kamayura, Yudjá, Waurá e Tapayuna e 2.957 pessoas de acordo com o censo populacional de 2013. A população desse território é jovem, representada por 38,2\% menores de 15 anos e apenas 5,4\% maiores de 60 anos. Constata-se uma distribuição similar entre os sexos, sendo $51 \%$ do sexo feminino e um predomínio da etnia Kaiabi, representando $43 \%$ da população do território.

Foi realizado um estudo descritivo de série temporal, com dados secundários obtidos do banco de dados do Projeto Xingu da UNIFESP, de 2004-2006, e do Sistema Local de Informação de Saúde (SLIS) do DSEI Xingu, de 2006-2013. Foram utilizados instrumentos e fichas para a obtenção de dados da atenção individual e coletiva de saúde bucal. Para o controle e avaliação da atenção à saúde bucal prestada, foram definidos pela FUNASA e pelo Ministério da Saúde alguns indicadores a ser utilizados pela equipe de saúde para acompanhar a dinâmica do atendimento realizado, o impacto e os resultados do trabalho odontológico na população indígena 21. Foram selecionados os seguintes indicadores, todos aplicados para a área relativa ao baixo, médio e leste Xingu: (1) cobertura de primeira consulta odontológica programática para aferir o acesso ao tratamento odontológico 22. Esse indicador mostra o percentual de pessoas que receberam uma primeira consulta odontológica programática, com a finalidade de diagnóstico e, necessariamente, a elaboração de um plano preventivo-terapêutico, para as necessidades detectadas e posterior realização do tratamento odontológico restaurador no âmbito da atenção básica; (2) tratamento odontológico básico concluído para quantificar o percentual de pacientes que teve tratamento concluído e está, portanto, livre de cárie dentária e doença periodontal em relação ao que realizou consulta odontológica programática; (3) proporção de exodontia em relação aos procedimentos preconizada pelas diretrizes de pactuação de objetivos, metas e indicadores de 2013-2015 do Ministério da Saúde com o intuito de representar o percentual de extrações dentárias realizadas em relação ao total de procedimentos clínicos individuais preventivos e curativos 15; (4) cobertura média mensal da escovação dental supervisionada que corresponde à média da ação coletiva de escovação dental supervisionada preconizada pelas diretrizes de pactuação de objetivos, metas e indicadores de 2013-2015 do Ministério da Saúde com o intuito de verificar o acesso ao fluoreto por meio da escovação com creme dental de uma população pelo número de participantes da escovação dental supervisionada mensalmente em relação à população 15.

Os dados foram processados no programa Microsoft Excel 2007 (Microsoft Corp., Estados Unidos) e, para organizar a apresentação dos dados, foram utilizados gráficos e tabelas que expressam a distribuição de frequências absolutas e relativas. Numa análise de série temporal em área de reduzida mudança demográfica, os indicadores selecionados são muito úteis para determinar se a intervenção 
está ampliando ou restringindo a cobertura da escovação dental supervisionada, o acesso ao tratamento odontológico programático e sua conclusão. Variações amplas não são esperadas e denotam problemas para assegurar os recursos necessários e mantê-los em operação.

O projeto de pesquisa que deu origem ao presente estudo foi submetido à aprovação pelo Comitê de Ética em Pesquisa (COEP) da Faculdade de Saúde Pública/USP (CAAE no 40968815.0.0000.5421) e pela Comissão Nacional de Ética em Pesquisa (CONEP), sob o parecer no 1.004.479. Foram seguidas as recomendações para pesquisas que envolvem populações indígenas, inclusas nas Resoluções no 196/1996 e no 466/2012, do Conselho Nacional de Saúde (CNS).

\section{Resultados}

O trabalho desenvolvido no DSEI Xingu, no período analisado, abrangeu ações de promoção e proteção de saúde, recuperação e reabilitação. Os dados relativos aos procedimentos coletivos e individuais foram consolidados mensalmente. Os procedimentos individuais foram divididos em preventivos, restauradores, endodônticos, periodontais e cirúrgicos. Os coletivos contemplaram procedimentos relacionados à educação em saúde, higiene bucal supervisionada, aplicação tópica de flúor e controle de distribuição de escova, creme e fio dental. A obtenção dos dados e a construção dos indicadores foram realizadas em julho e agosto de 2015. Foram analisados 360 relatórios mensais, consolidados em trinta planilhas anuais.

Na Tabela 1, são apresentados os indicadores cobertura de primeira consulta odontológica programática e tratamento odontológico básico concluído, referentes aos anos 2006-2013. Nos anos de 2004 e 2005, não constavam informações sobre primeira consulta odontológica programática, impossibilitando a construção de tais indicadores.

A cobertura da primeira consulta odontológica foi superior a $60 \%$ em todos os anos analisados, exceto 2009 e 2010 com uma cobertura de 44,7\% e 53,4\%, respectivamente. Os resultados apresentados mostraram um declínio do acesso ao tratamento odontológico programático a partir de 2008 , sendo restabelecido somente a partir de 2011. A Figura 1 ilustra as diferenças, indicando uma variação entre $44,7 \%$ a $71,3 \%$ com uma taxa média para o período de $63,4 \%$

Referentemente ao tratamento odontológico básico concluído, encontra-se um aumento entre os anos 2006 e 2008, de 44,9\% para 79,9\%. A taxa média foi de 59,8\% para o período de $2006-2013$. No ano de 2009, constataram-se o segundo maior indicador de tratamento odontológico concluído e o menor valor de cobertura de primeira consulta odontológica em todo o período analisado. De 20102013 , os valores variaram de $52,8 \%$ a $65,3 \%$.

Tabela 1

Indicadores de cobertura de primeira consulta odontológica programática e tratamento odontológico concluído. Parque Indígena Xingu, Brasil, no período de 2006-2013.

\begin{tabular}{|c|c|c|c|c|c|c|c|c|c|}
\hline Indicador & Medida & 2006 & 2007 & 2008 & 2009 & 2010 & 2011 & 2012 & 2013 \\
\hline $\begin{array}{l}\text { Cobertura de primeira consulta odontológica } \\
\text { programática }\end{array}$ & $\begin{array}{l}\text { Total de primeiras consultas odontológicas } \\
\text { programáticas realizadas em determinado } \\
\text { local e período/População total em } \\
\text { determinado local e período x } 100\end{array}$ & 67,4 & 69,9 & 60,0 & 44,7 & 53,4 & 71,2 & 70,2 & 70,6 \\
\hline Tratamento odontológico básico concluído & $\begin{array}{c}\text { Total de pessoas que concluíram } \\
\text { o tratamento odontológico básico } \\
\text { em determinado local e período/Total } \\
\text { de pessoas que realizaram consulta } \\
\text { odontológica programada em determinado } \\
\text { local e período x } 100\end{array}$ & 44,9 & 48,1 & 79,9 & 66,7 & 52,8 & 65,3 & 63,3 & 57,7 \\
\hline
\end{tabular}

Fonte: Distrito Sanitário Especial Indígena Xingu/Projeto Xingu. 
Valores de cobertura de primeira consulta odontológica programática da atenção à saúde bucal. Parque Indígena Xingu, Brasil, no período de 2006-2013.

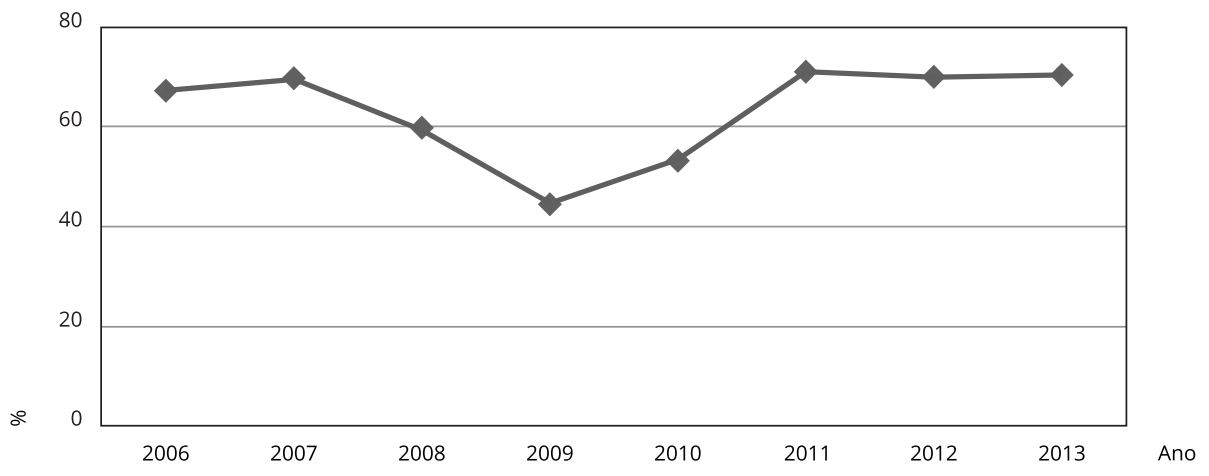

Fonte: Sistema Local de Informação de Saúde, Distrito Sanitário Especial Indígena Xingu/Projeto Xingu.

O total de exodontias em relação ao total de procedimentos é um indicador que pode refletir a priorização das ações em curativas, preventivas ou cirúrgicas. A Tabela 2 mostra os valores referentes ao período de 2004-2013 obtidos para extrações dentárias, procedimentos clínicos individuais preventivos e curativos e proporção de exodontia. Os valores mínimo e máximo de extrações dentárias foram respectivamente, 170 e 604 , com uma média de 499,5 no período. No ano de 2009 , foram encontrados o menor número de extrações e o segundo menor número de procedimentos preventivos e curativos. Os valores mínimo e máximo de procedimentos preventivos e curativos foram, respectivamente, 2.481 e 12.507, com uma média de 7.346,2 no período.

O indicador proporção de exodontia expressa seu valor mais alto no ano de 2004. Há uma queda brusca entre 2004 e 2005 de 24,3\% para 10,7\%. Esse indicador mantém uma queda importante com o passar dos anos chegando a 3,8\% em 2011, com uma taxa média de $8,6 \%$ no período de análise. A Figura 2 ilustra os valores encontrados em cada ano.

$\mathrm{O}$ indicador escolhido para ilustrar as ações coletivas foi cobertura média mensal da escovação dental supervisionada. A Tabela 3 mostra o número de participantes da escovação dental supervisionada e os valores do indicador para o período de 2004-2013 que indica uma variação entre 1,2 a $23,3 \%$, com uma taxa média para o período de $10,8 \%$.

\section{Discussão}

A Organização Pan-Americana da Saúde (OPAS) afirma que a melhoria da qualidade de vida da população se justifica na melhoria do acesso, resolução das necessidades em saúde, estímulo à participação social e redução das desigualdades 23 . Iniquidades entre indígenas e não indígenas, relacionadas ao acesso a serviços de atenção à saúde bucal e métodos preventivos regulares são evidentes e deixam clara a vulnerabilidade dessas populações, inclusive em relação à cárie dentária e suas complicações 5,24. As intervenções em saúde introduzidas sem um planejamento adequado, que não possibilitem a universalização e a equidade, têm exercido o indesejável efeito de ampliar as desigualdades em saúde 25,26.

Em relação ao tratamento odontológico básico concluído, verificaram-se valores mais elevados no DSEI Xingu no período sob análise, em comparação aos valores divulgados na síntese dos indicadores de vigilância em saúde indígena entre os anos 2004-2010. Enquanto a taxa média para a população xinguana foi $59,8 \%$, para a população indígena em geral foi 16,7\%, variando de $12,8 \%$ em 2004 para $23 \%$ em 2010 27. As elevadas taxas encontradas nesse indicador podem estar relacionadas ao traba- 


\section{Tabela 2}

Números totais de extrações dentárias, números totais de procedimentos clínicos individuais preventivos e curativos e indicadores de proporção de exodontia em relação aos procedimentos. Parque Indígena Xingu, Brasil, no período de 2004-2013.

\begin{tabular}{|c|c|c|c|c|c|c|c|c|c|c|}
\hline Medida & 2004 & 2005 & 2006 & 2007 & 2008 & 2009 & 2010 & 2011 & 2012 & 2013 \\
\hline Número total de extrações dentárias & 602 & 549 & 604 & 546 & 466 & 170 & 481 & 480 & 512 & 585 \\
\hline $\begin{array}{l}\text { Número total de procedimentos clínicos } \\
\text { individuais preventivos e curativos }\end{array}$ & 2.481 & 5.134 & 4.765 & 7.115 & 10.353 & 4.215 & 7.076 & 12.507 & 10.647 & 9.169 \\
\hline $\begin{array}{l}\text { Proporção de exodontia em relação aos } \\
\text { procedimentos }\end{array}$ & 24,3 & 10,7 & 12,7 & 7,7 & 4,5 & 4,0 & 6,8 & 3,8 & 4,8 & 6,4 \\
\hline
\end{tabular}

Fonte: Distrito Sanitário Especial Indígena Xingu/Projeto Xingu.

\section{Figura 2}

Valores de proporção de exodontia em relação aos procedimentos individuais da atenção à saúde bucal. Parque Indígena Xingu, Brasil, no período de 2004-2013.

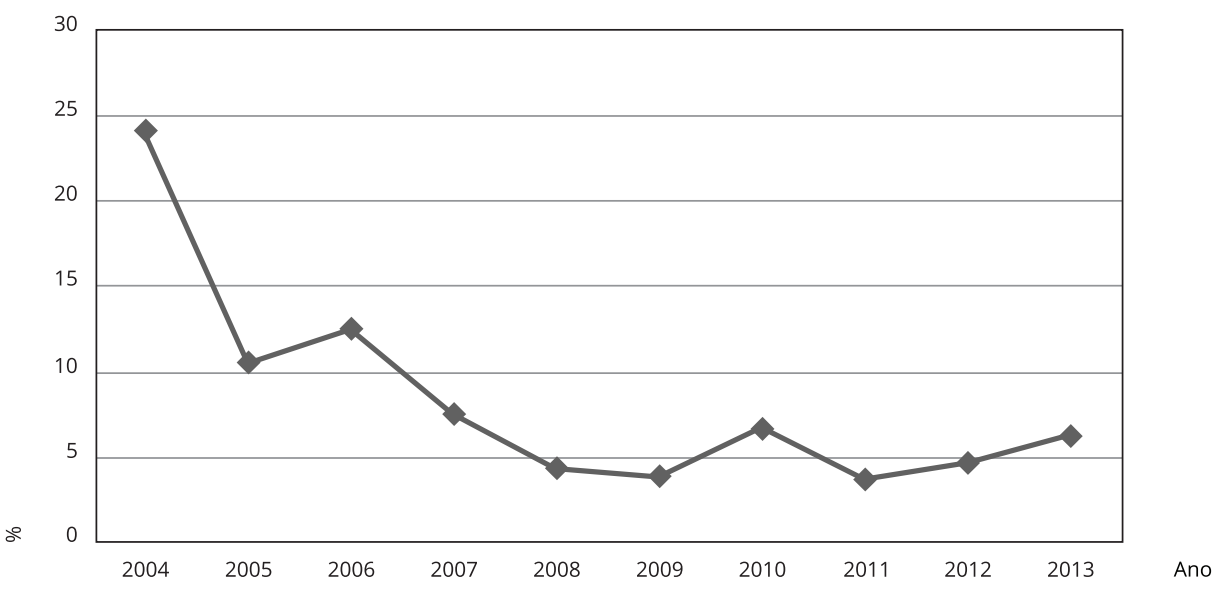

Fonte: Sistema Local de Informação de Saúde, Distrito Sanitário Especial Indígena Xingu/Projeto Xingu.

lho diferenciado construído historicamente pelo Projeto Xingu na região. O Projeto Xingu é o mais longo programa de extensão e pesquisa em saúde indígena, com mais de 50 anos de existência, sendo uma exceção no país. O estabelecimento de parcerias com a FORP-USP possibilitou a colaboração de professores e alunos para a estruturação do programa de atenção à saúde bucal em todas as etapas e melhorou o aporte de insumos, especialmente de material de higiene bucal, fornecidos pelo Projeto Pro Nativos e utilizados no trabalho de campo no PIX. Com isso, foram possíveis a realização de inquéritos epidemiológicos, o fortalecimento do trabalho preventivo nas escolas indígenas, a ampliação do acesso ao flúor tópico, a formação continuada de indígenas para atuarem na saúde bucal, a capacitação da equipe para o trabalho intercultural e a participação do controle social 18 .

Outro aspecto positivo foi a redução na proporção de exodontia em relação aos procedimentos individuais. Isso pode estar associado à extensão de cobertura propiciada pela parceria com a FORP-USP. O comportamento desse indicador pode revelar o pouco acesso da população aos serviços de saúde bucal, gerando uma demanda acumulada de exodontias, a falta de investimento em ações preventivas em longo prazo e o modelo de atenção hegemônico 28 . Importante perceber que a melhora 
Tabela 3

Números totais de participantes de ação coletiva de escovação dental supervisionada e indicadores de média da ação coletiva de escovação dental supervisionada. Parque Indígena Xingu, Brasil, no período de 2004-2013.

\begin{tabular}{|c|c|c|c|c|c|c|c|c|c|c|}
\hline Medida & 2004 & 2005 & 2006 & 2007 & 2008 & 2009 & 2010 & 2011 & 2012 & 2013 \\
\hline $\begin{array}{l}\text { Número total de participantes de ação coletiva de } \\
\text { escovação dental supervisionada }\end{array}$ & 1.652 & 2.052 & 3.657 & 3.643 & 2.876 & 863 & 385 & 6.731 & 3.961 & 8.252 \\
\hline $\begin{array}{l}\text { Média da ação coletiva de escovação dental } \\
\text { supervisionada }\end{array}$ & 6,5 & 8,0 & 12,7 & 12,3 & 9,9 & 2,9 & 1,2 & 19,3 & 11,5 & 23,3 \\
\hline
\end{tabular}

Fonte: Distrito Sanitário Especial Indígena Xingu/Projeto Xingu.

em tal indicador parece estar mais relacionada ao aumento do número absoluto de procedimentos preventivos e curativos do que à diminuição do número absoluto de extrações dentárias. Ainda assim, o indicador evidencia a priorização dos procedimentos preventivos e curativos com o decorrer dos anos.

As condições de acesso refletem as características da oferta de serviços de acordo com as necessidades e demandas da população, apesar de não traduzir, num primeiro momento, em reversão do modelo de atenção e não analisar a resolutividade dos serviços ${ }^{29}$. Analisando a cobertura da população com primeira consulta odontológica programática é possível identificar a necessidade de implantação de medidas para ampliação do acesso aos serviços odontológicos e a avaliação do perfil de atendimento desse serviço 22 . Nota-se uma cobertura favorável de primeira consulta odontológica programática no PIX, sendo maior que $60 \%$ na maioria dos anos analisados, mostrando a busca pela ampliação do acesso à assistência odontológica programática. O declínio desse indicador a partir de 2008 e seu o restabelecimento em 2011 podem estar relacionados ao período de encerramento das parcerias e da mudança da gestão do SASI-SUS, influenciando negativamente os indicadores de saúde bucal. Com a mudança da gestão, os DSEI passaram por problemas estruturais e, apesar do aumento significativo de orçamento e recursos humanos, o que se observou foi a não priorização de ações que assegurassem a atenção básica à saúde como o investimento em saneamento básico, na qualificação de profissionais, no controle e fiscalização das ações e no planejamento efetivo ${ }^{30}$. No DSEI Xingu não houve aumento de profissionais da área de odontologia, apesar de o número de enfermeiros ter triplicado após a criação da SESAI. Os processos de compras de kits de higiene bucal, materiais permanentes e insumos para a realização do tratamento restaurador atraumático, ficaram centralizados em Brasília, e durante todo o ano de 2010 não foram disponibilizados.

O único estudo encontrado, em povos indígenas, que utilizou um indicador de acesso aos serviços de saúde bucal foi o realizado no PIX e indica um aumento significativo dos valores entre 2004 e 2006. Embora os indicadores tenham sido calculados de forma diferente deste estudo, é perceptível o aumento do número de atendimentos durante o período, em consequência da maior disponibilidade de recursos humanos 18. Estudos com populações não indígenas, em bases de dados dos municípios de Belo Horizonte (Minas Gerais), Amaral Ferrador (Rio Grande do Sul) e João Pessoa (Paraíba), relataram melhora do indicador de primeira consulta odontológica programática no período analisado (1999-2010), em decorrência da ampliação do número de profissionais de saúde bucal contratados pela estratégia saúde da família, a favorável incorporação de redes de cuidados em saúde bucal e a capacitação de profissionais para o preenchimento de informações 31,32,33.

A integralidade da atenção à saúde bucal, proposta pela Política Nacional de Saúde Bucal, pode ser garantida com a oferta adequada de ações intersetoriais, individuais e coletivas, que contemplem as dimensões biológica, psicológica e social e sejam articuladas com os outros níveis de atenção. Um dado preocupante para a integralidade da atenção foi o fraco desempenho do indicador de escovação supervisionada em alguns anos analisados. Uma questão fundamental na implantação dos programas de saúde bucal nos DSEI é a necessidade de priorizar a dimensão preventiva e educativa, considerando o contexto sociocultural, político e econômico de cada comunidade e o acesso ao flúor 34 . A média da ação coletiva de escovação dental supervisionada foi o indicador que apresentou maior variação entre todos os anos, de $1,2 \%$ a 23,3\%. Os piores valores foram encontrados nos anos 2009 e 2010; 
um resultado que pode estar associado ao encerramento da parceria com o Projeto Pro Nativos que, entre outros aspectos, assegurava acesso a material de higiene bucal. Somente em 2011, esse material é novamente disponibilizado pela SESAI, possibilitando o aumento dos valores desse indicador.

O número de estudos científicos sobre programas de saúde bucal para povos indígenas no Brasil ainda é pequeno e algumas experiências descritas, como nos Enawenê-nawê e nos Xavante de Pimentel Barbosa, refletem sobre a necessidade de priorizar a dimensão preventiva, a formação de indígenas para atuação na saúde bucal, o acesso continuado aos materiais de higiene bucal e o estabelecimento de parcerias com instituições não governamentais 25,35. Alguns estudos internacionais sobre programas de saúde bucal para povos indígenas têm reforçado a necessidade de observar algumas estratégias para que um programa de saúde bucal seja bem-sucedido como: a necessidade de estratégias e abordagens de promoção de saúde por ciclos da vida, a garantia do acesso a materiais para educação em saúde de cuidadores e gestantes, a formação de profissionais indígenas para atuação na saúde bucal, a atuação multiprofissional e a abordagem de fator comum e a integração da atenção à saúde com as práticas tradicionais $8,10,11,12,13$. Projetos que surgiram de preocupações, necessidades e envolvimento direto da comunidade tiveram melhores resultados em longo prazo 8,12.

Importante destacar as várias estratégias positivas realizadas no PIX com a equipe de saúde, particularmente com os agentes indígenas de saúde, para a consolidação de ações intersetoriais com a educação e para o aumento da cobertura de escovação supervisionada. Vários fatores relacionados a recursos logísticos, humanos e culturais impossibilitaram a ampliação do acesso. Uma das bases da estratégia era a abordagem interdisciplinar para solucionar alguns dos problemas encontrados.

Um exemplo foi inserir a escovação supervisionada e a aplicação tópica de flúor durante as etapas de imunização para ampliar a cobertura de acesso ao flúor. O programa de imunização se mostrou oportuno para o trabalho preventivo em saúde bucal por causa da sua periodicidade, alta cobertura, organização logística, alta adesão e possibilidade de acompanhamento de profissionais de forma regular e interdisciplinar, mediante a abordagem ampla do cuidado. A imunização traz uma "memória positiva" para a comunidade, pois foi por intermédio do programa inicial do Projeto Xingu que a mortalidade diminuiu e permanece na memória de muitas lideranças, que relatam sua prioridade e importância. Tal estratégia não foi realizada nos anos de 2009 e 2010, refletindo no indicador correspondente.

Os resultados deste estudo mostram que os programas de intervenção na saúde de populações indígenas se tornam cada vez mais necessários à melhoria dos serviços de atenção à saúde indígena e requerem monitoramento e adoção de estratégias diferenciadas. Uma limitação deste estudo se refere à impossibilidade de uma análise mais aprofundada dos indicadores por variáveis importantes para compreensão das especificidades socioculturais envoltas no processo saúde-doença dessas populações, como polo base, etnia, sexo e grupo etário. Durante a análise dos dados, verificou-se que algumas informações presentes nos instrumentos de registro do Sistema de Informação da Atenção à Saúde Indígena (SIASI) não foram registradas pelos profissionais de saúde, não estando disponíveis nos relatórios consolidados, fonte de dados do presente estudo. Isso gera reflexão sobre a importância da capacitação e acompanhamento sistemático do preenchimento adequado desses instrumentos para a qualidade e completude dos dados, possibilitando uma avaliação adequada aos serviços de saúde. Cumpre ainda destacar as limitações do uso de dados secundários derivados dos sistemas de informação utilizados, o que vai ao encontro do estudo de Sousa et al. 36 sobre a necessidade de ajustes estruturais no SIASI para monitoramento das condições de saúde nas comunidades indígenas. Ainda que o SIASI apresente limitações desde a concepção à operacionalização 36, há que se reconhecer que apresenta potencialidades para o uso em pesquisas avaliativas e para os serviços de saúde.

\section{Conclusões}

Este estudo tem implicações importantes para a implementação de programas de saúde bucal em áreas indígenas e relata estratégias de intervenções e parcerias para melhorar a oferta e organização dos serviços, a equidade e o acesso da população indígena à saúde bucal, buscando diminuir os entraves burocráticos e as iniquidades de saúde. Descreve também caminhos para a busca da integralidade da atenção pela participação da própria comunidade e articulação com outros setores, por meio de estratégias sensíveis às especificidades locais. 
O estudo também expõe as dificuldades e a insensibilidade da gestão do SASI-SUS para assegurar a continuidade de um programa de atenção à saúde bucal que vinha contribuindo para mudanças positivas nos indicadores e nas condições de saúde bucal durante seu período de atividade. A descontinuidade do programa e o período de mudança da gestão do SASI-SUS trouxeram problemas para a saúde bucal da população do DSEI Xingu. Apesar de avanços durante o período estudado, a piora de alguns indicadores de saúde bucal nos últimos anos coincide também com a piora de outros indicadores de saúde. Isso produz reflexão sobre a influência direta de estratégias e decisões tomadas por gestores na saúde dos povos indígenas e alerta indígenas e indigenistas para que estejam constantemente atentos em seus movimentos e para que a conquista de um subsistema específico de atenção diferenciada de saúde não seja perdida.

A produção de indicadores para o uso dos serviços de atenção à saúde indígena do DSEI Xingu é uma importante contribuição. Diante dos resultados obtidos, pode-se concluir que o acesso à saúde bucal tem mostrado uma boa cobertura, apesar do constatado período de queda. $\mathrm{O}$ indicador de tratamento odontológico básico concluído apresenta um alto percentual comparado com a população indígena como um todo no mesmo período do estudo.

As quedas encontradas no indicador de exodontia podem estar relacionadas ao apoio da FORP-USP até 2008. O final desse apoio e o período de mudança de gestão influenciaram negativamente todos os indicadores de saúde bucal do DSEI Xingu. Apesar de os indicadores de escovação supervisionada revelarem a necessidade da priorização das ações preventivas, o que se verificou foi o predomínio de um modelo assistencialista, em que a alta necessidade de tratamento é priorizada em relação às ações de prevenção de doenças e promoção da saúde. Não obstante, a ação multiprofissional durante as visitas nas aldeias e nas etapas de imunização, como forma de ampliar a média de escovação dental supervisionada, mostrou-se positiva. A atuação multiprofissional ampliou a relevância das ações de promoção de saúde bucal tanto para os profissionais de saúde quanto para as comunidades

Os desafios relacionados à integralidade da atenção e à articulação com as outras instâncias do SUS, com a participação efetiva dos indígenas, devem ser pensados para a continuidade desse programa, mediante o diálogo e a negociação. Integrar instituições de ensino e pesquisa na atenção à saúde, de forma sustentável, pode ser um dos possíveis caminhos para diminuir as iniquidades do acesso à saúde e a efetividade de programas voltados à população indígena que se torna cada vez mais vulnerável às doenças e às interferências políticas que permeiam a gestão dos serviços de saúde.

\section{Colaboradores}

P. N. Lemos e P. C. Narvai contribuíram na idealização e participação em todas as etapas do estudo. A. C. Guisilini e L. B. Hirooka colaboraram na transcrição, confiabilidade e análise dos dados. D. A. Rodrigues e P. Frazão contribuíram na realização da revisão final do manuscrito. Todos os autores participaram do planejamento do estudo, revisão e aprovação da versão final do manuscrito.

\section{Agradecimentos}

Nossos agradecimentos ao Conselho Distrital de Saúde Indígena do Xingu, às lideranças indígenas do Xingu, ao DSEI Xingu, ao Projeto Xingu, Universidade Federal de São Paulo e à Faculdade de Saúde Pública, Universidade de São Paulo, por possibilitarem este estudo.

\section{Referências}

1. Paim JS. Bases conceituais da reforma sanitária brasileira. In: Fleury S, organizador. Saúde e democracia: a luta do Cebes. São Paulo: Lemos Editorial; 1997. p. 11-24.

2. Narvai PC, Frazão P. Saúde bucal no Brasil: muito além do céu da boca. Rio de Janeiro: Editora Fiocruz; 2008.

3. Alves Filho P, Santos RV, Vettore MV. Fatores associados à cárie dental e doença periodontal em indígenas na América Latina: revisão sistemática. Rev Panam Salud Pública 2014; 35:67-77.

4. Arantes R. Saúde bucal dos povos indígenas no Brasil: panorama atual e perspectivas. In: Coimbra Jr. CEA, Santos RV, Escobar AL, organizadores. Epidemiologia e saúde dos povos indígenas no Brasil. Rio de Janeiro: Abrasco/ Editora Fiocruz; 2003. p. 49-72.

5. Ha DH, Xiangqun J, Cecilia MG, Jason A, Do LG, Jamieson, LM. Social inequality in dental caries and changes over time among Indigenous and non-Indigenous Australian children. Aust N Z J Public Health 2016; 40:542-7. 
6. Ha DH. Oral health of Australian Indigenous children compared to non-Indigenous children enrolled in school dental services. Aust Dent J 2014; 59:395-400.

7. Campbell MA, Hunt J, Walker D, Williams R. The oral health care experiences of NSW Aboriginal Community Controlled Health Services. Aust N Z J Public Health 2015; 39:21-5.

8. Patel J, Hearn L, Gibson B, Slack-Smith LM. International approaches to Indigenous dental care: what can we learn? Aust Dent J 2014; 59:439-45.

9. Jamieson LM, Divaris K, Parker EJ, Lee JY. Oral health literacy comparisons between Indigenous Australians and American Indians. Community Dent Health 2013; 30:52-7.

10. Parker EJ, Jamieson LM, Broughton J, Albino J, Lawrence HP, Roberts-Thomson K. The oral health of Indigenous children: a review of four nations. J Paediatr Child Health 2010; 46:483-6.

11. Broughton J. An oral health intervention for the Maori indigenous population of New Zealand: oranga niho Maori (Maori oral health) as a component of the undergraduate dental curriculum in New Zealand. Int Dent J 2010; 60(3 Suppl 2):223-8.

12. Lawrence, HP. Oral health interventions among Indigenous populations in Canada. Int Dent J 2010; 60(3 Suppl 2):229-34.

13. Riedy C. A dental intervention with an Alaskan Native population: lessons learned. Int Dent J 2010; 60(3 Suppl 2):241-4.

14. Secretaria Especial de Saúde Indígena, Ministério da Saúde. Diretrizes do componente indígena da Política Nacional de Saúde Bucal. Brasília: Ministério da Saúde; 2011.

15. Departamento de Articulação Interfederativa, Secretaria de Gestão Estratégica, Ministério da Saúde. Caderno de diretrizes, objetivos, metas e indicadores: 2013-2015. Brasília: Ministério da Saúde; 2013. (Série Articulação Interfederativa, 1).

16. Moreira RS, Nico LS, Tomita NE, Ruiz T. A saúde bucal do idoso brasileiro: revisão sistemática sobre o quadro epidemiológico e acesso aos serviços de saúde bucal. Cad Saúde Pública 2005; 21:1665-75.

17. Coeli CM. Sistemas de Informação em Saúde e uso de dados secundários na pesquisa e avaliação em saúde. Cad Saúde Colet (Rio J.) 2010; 18:335-6.

18. Lemos PN, Hirooka LB, Nunes SAC, Arantes R, Mestriner SF, Mestriner Jr. W. O modelo de atenção a saúde bucal no Médio e Baixo Xingu: parcerias, processos e perspectivas. Ciênc Saúde Coletiva 2010; 15 Suppl 1:1449-56.

19. Martins AL. Política de saúde indígena no Brasil: reflexões sobre o processo de implementação do Subsistema de Atenção à Saúde Indígena [Dissertação de Mestrado]. Brasília: Escola Nacional de Saúde Pública Sergio Arouca, Fundação Oswaldo Cruz; 2013.

20. Tamaki EM, Tanaka OY, Felisberto E, Alves CKA, Drumond Jr. M, Bezerra LCA, et al. Metodologia de construção de um painel de indicadores para o monitoramento e a avaliação da gestão do SUS. Ciênc Saúde Coletiva 2012; 17:839-49.
21. Fundação Nacional de Saúde. Diretrizes para a atenção à saúde bucal nos Distritos Sanitários Especiais Indígenas: manual técnico. Brasília: Fundação Nacional da Saúde; 2007.

22. Ministério da Saúde. Portaria no 493, de 10 de março de 2006. Aprova a Relação de Indicadores da Atenção Básica - 2006, cujos indicadores deverão ser pactuados entre municípios, estados e Ministério da Saúde. Diário Oficial da União 2006; 13 mar.

23. Conselho Nacional de Saúde. 3a Conferência Nacional de Saúde Bucal. Saúde Bucal: acesso e qualidade, superando a exclusão social. Relatório final. Brasília: Ministério da Saúde; 2005.

24. Arantes R, Santos RV, Frazão P. Oral health in transition: the case of Indigenous peoples from Brazil. Int Dent J 2010; 60(3 Suppl 2):235-40.

25. Antunes JLF, Narvai PC. Políticas de saúde bucal no Brasil e seu impacto sobre as desigualdades em saúde. Rev Saúde Pública 2010; 44:360-5.

26. Albino JE, Orlando VA. Promising directions for caries prevention with American Indian and Alaska Native children. Int Dent J 2010; 60(3 Suppl 2):216-22.

27. Fundação Nacional de Saúde. Vigilância em saúde indígena: síntese dos Indicadores 2010. Brasília: Fundação Nacional de Saúde; 2010.

28. Fernandes LSP, Peres MA. Associação entre atenção básica em saúde bucal e indicadores socioeconômicos municipais. Rev Saúde Pública 2005; 39:930-6.

29. Barreto Junior IFB, Ferreira MP, Silva ZP. Pesquisa de condições de vida 2006: acesso aos serviços odontológicos: uma avaliação em nível nacional. Ciênc Saúde Coletiva 2008; 22:5-18.

30. Liebgott RA. Atenção à saúde indígena no Brasil: uma realidade devastadora. In: Rangel LH, organizador. Violência contra os povos indígenas no Brasil: dados de 2013. Brasília: Conselho Indigenista Missionário; 2014. p. 20-2.

31. Cruz DF, Prado RL, Valença AMG, Machado LS. A Linha do Cuidado em Saúde Bucal no município de João Pessoa: uma análise de indicadores. Pesqui Bras Odontopediatria Clín Integr 2011; 11:291-5.

32. Mendes SR, Esteves RSS, Oliveira ACB, Abreu MHNG. Avaliação de indicadores de saúde bucal na atenção primária em um município de grande porte brasileiro de 1998 a 2010. SaBios (Campo Mourão Online) 2013; 8:74-82.

33. Teixeira ND, Facchini LA, Castilho ED. Avaliação da evolução da demanda de saúde bucal através do uso de sistemas de informação em saúde. Revista de Enfermagem e Saúde 2011; 1:50-9.

34. Arantes R. Saúde bucal dos povos indígenas no Brasil e o caso dos Xavante de Mato Grosso [Tese de Doutorado]. Rio de Janeiro: Escola Nacional de Saúde Pública, Fundação Oswaldo Cruz; 2005

35. Arantes R, Frazão P. Cárie dentária entre os povos indígenas do Brasil: implicações para os programas de saúde bucal. Tempus (Brasília) 2013; 7:169-80.

36. Sousa MC, Scatena JHG, Santos RV. O Sistema de Informação da Atenção à Saúde Indígena (SIASI): criação, estrutura e funcionamento. Cad Saúde Pública 2007; 23:853-61. 


\section{Abstract}

In 2011, the Brazilian National Oral Health Policy redefined its model of care for indigenous peoples, recommending the use of epidemiology and followup of the impact of activities using adequate indicators. The current study aimed to analyze trends in these indicators, proposed by the Brazilian Ministry of Health, in the Xingu Indigenous Park, Brazil, from 2004 to 2013. This was a quantitative study using secondary data from the Xingu Special Indigenous Health District and the Xingu Project of the Federal University of São Paulo. The coverage rate for first dental visit exceeded $60 \%$ in all the years analyzed except 2009 and 2010 (44.7\% and $53.4 \%$, respectively). The basic dental treatment indicator showed a significant increase, from 44.9\% to 79.9\%, between 2006 and 2008. The proportion of tooth extractions decreased from 24.3\% in 2004 to 3.8\% in 2011. Mean coverage of supervised collective toothbrushing showed the highest variation (1.2 to 23.3\%). Access to oral health showed good coverage, and the indicator for completed treatment showed a higher percentage when compared to other indigenous peoples during the same period. Better performance on the tooth extractions indicator may have been due to the change in focus of care through partnerships with universities, although the indicators for supervised toothbrushing suggest the need to prioritize preventive measures. Changes in indigenous healthcare management, with weakening or absence of partnerships, may have negatively influenced the program's indicators.

Health of Indigenous Peoples; Oral Health; Indicators of Health Services; South American Indians

\section{Resumen}

La Política Nacional de Salud Bucal redefinió en 2011 su modelo de atención para los pueblos indígenas, preconizando el subsidio de la epidemiologia y el seguimiento del impacto de las acciones mediante indicadores adecuados. El objetivo de este estudio fue analizar la evolución de esos indicadores, propuestos por el Ministerio de la Salud, en el Parque Indígena de Xingu, Brasil, durante el periodo de 2004 a 2013. Se trata de una investigación de enfoque cuantitativo, con el uso de datos secundarios del Distrito Sanitario Especial Indígena Xingu y del Proyecto Xingu, de la Universidad Federal de São Paulo. Se observó una cobertura de primera consulta odontológica programática mayor que un $60 \%$ en todos los años analizados, excepto en 2009 y 2010, cuyas coberturas fueron de un $44,7 \%$ y un $53,4 \%$, respectivamente. El indicador de tratamiento odontológico básico concluido presentó un aumento significativo, de un 44,9\% a un 79,9\%, entre 2006 y 2008. La proporción de exodoncias en el conjunto de los procedimientos disminuyó de un 24,3\% en 2004 a un 3,8\% en 2011. La cobertura de la media de la acción colectiva de cepillado dental supervisado obtuvo la mayor variabilidad $(1,2$ a $23,3 \%)$ durante el período analizado. El acceso a la salud bucal mostró una buena cobertura y el indicador de tratamiento concluido presentó un porcentaje más elevado, en comparación con otros pueblos indígenas durante el mismo periodo. El mejor desempeño del indicador de exodoncias puede producirse del cambio en el enfoque asistencial, posibilitado por colaboraciones con universidades, aunque los indicadores de cepillado supervisado indiquen que sea necesario priorizar acciones preventivas. Cambios en la gestión de la salud indígena, con fragilización o ausencia de colaboraciones, pueden haber influenciado negativamente los indicadores del programa.

Salud de Poblaciones Indígenas; Salud Bucal; Indicadores de Servicios; Indios Sudamericanos
Recebido em 09/Mai/2017

Versão final reapresentada em 08/Out/2017

Aprovado em 18/Out/2017 